\title{
High-dose Somatostatin Analogues for Progressive Neuroendocrine Tumours
}

\author{
Anna J Sharp, ${ }^{1}$ Aimee R Hayes ${ }^{2}$ and Ashley Grossman ${ }^{1,2}$ \\ 1. Green Templeton College, University of Oxford, Oxford, UK; 2. Neuroendocrine Tumour Unit, European Neuroendocrine Tumour Society \\ (ENETS) Centre of Excellence, Royal Free Hospital, London, UK
}

$\mathrm{N}$ euroendocrine tumours are a heterogeneous group of neoplasms with a variety of treatment options, but studies suggest they should in most cases, if they are not curable surgically, be treated initially with monthly long-acting somatostatin analogues. New data recently published suggest that if patients show progression on the current analogues at the recommended doses, increasing the dose or frequency of these analogues may provide a further therapeutic effect, although proper randomised trials will be necessary to confirm this effect.

\section{Keywords}

High-dose, neuroendocrine tumours (NETs), lanreotide, octreotide, somatostatin analogues

Disclosures: Anna J Sharp, Aimee R Hayes and Ashley Grossman have no financial or non-financial relationships or activities to declare in relation to this article

Review Process: Double-blind peer review. Compliance with Ethics: This article is an opinion piece and does not report on new clinical data, or any studies with human or animal subjects performed by any of the authors.

Authorship: The named authors meet the International Committee of Medical Journal Editors (ICMJE) criteria for authorship of this manuscript, take responsibility for the integrity of the work as a whole, and have given final approval for the version to be published.

Access: This article is freely accessible at

touchENDOCRINOLOGY.COM

(C) Touch Medical Media 2020

Received: 15 April 2020

Accepted: 8 June 2020

Published Online: 13 August 2020

Citation: European Endocrinology. 2020;16(2):93-5

Corresponding Author: Ashley Grossman, Neuroendocrine Tumour Unit, Royal Free Hospital,

Pond Street, Hampstead, London NW3 2QG, UK.

E: ashley.grossman@ocdem.ox.ac.uk

Support: No funding was received for

the publication of this article.
Neuroendocrine tumours (NETS) represent a heterogeneous group of neoplasms arising from the neuroendocrine cell system, primarily in the gastrointestinal or bronchial tracts. Well-differentiated, low-grade NETS (G1/G2) are often indolent in their behaviour; they may present with a lack of initial symptomatology, and can grow slowly. As a consequence, many cases are diagnosed at advanced stages, when curative resection is no longer viable. This, in concert with their rising incidence, ${ }^{1}$ highlights a need for improved systemic therapies to both delay tumour progression and reduce symptoms.

Somatostatin analogues (SSAS) are currently the first-line medical therapy for NETs, and provide a dual effect. First, their well-established anti-secretory actions diminish symptoms driven by hormonal hypersecretion, as is encountered in functional NETs. More recently, their anti-proliferative effects have been subject to increased focus, as a means of delaying disease progression in these patients. The primary mechanism by which SSAs exert their anti-tumoural effects is via G-protein-coupled somatostatin receptors (SSTR) expressed by NETs. Different SSTR subtypes are thought to be responsible for various anti-proliferative effects, with activation of phosphotyrosine phosphatases and modulation of the mitogen-activated protein (MAP) kinase pathway implicated in the potential mechanisms. ${ }^{2}$ SSAs may also exert indirect effects, such as the inhibition of mitogenic growth factors including insulin-like growth factor 1 (IGF-1), anti-angiogenic effects via SSTRs on endothelial cells and immunomodulatory effects. ${ }^{3}$

There are currently two SSAS approved for clinical use in patients with NETs: octreotide long-acting release (LAR) $30 \mathrm{mg}$, and lanreotide autogel $120 \mathrm{mg}$. Both medications are administered every 28 days, and are thought to act principally via SSTR-2. Their anti-proliferative effects in well-differentiated metastatic midgut NETs were evaluated in the phase III PROMID ${ }^{4}$ and CLARINET ${ }^{5}$ trials, and both were found to prolong progression-free survival (PFS) compared with placebo. While there exists a small increased propensity for biliary stone formation under SSAs, both drugs were generally well-tolerated, with severe adverse effects rare. For advanced NETs, the European Neuroendocrine Tumor Society (ENETS) guidelines thus recommend first-line treatment with SSAS in preference over molecular-targeted therapies and chemotherapy in order to preserve quality of life, unless tumours exhibit sinister attributes such as rapid progression, a high tumour burden or high proliferation index. ${ }^{6}$

Using SSAs at a higher, unconventional dosage is common in clinical practice, primarily to limit symptoms in syndromic patients. The ENETS guidelines also loosely suggest escalation of standard SSA therapy to high-dose SSAS for disease control, if the former proves ineffective - and yet, there is a paucity of quality evidence to support this recommendation, other than scattered small-scale studies. In one study of 12 patients with progressive midgut NETs transferred to octreotide pamoate $160 \mathrm{mg}$ every 2 weeks for 2 months and thereafter once monthly, 75\% showed tumour stabilisation for a median of 12 months.' However, this particular formulation is not currently available. Now, in a recently published study, Lamberti and colleagues have retrospectively surveyed the use of non-conventional high-dose SSAS (HD-SSAS) in 140 patients with advanced gastro-enteropancreatic NETS. ${ }^{8}$ All participants had radiologically confirmed 
progressive disease (as per RECIST [Response Evaluation Criteria in Solid Tumors] criteria), on a background of previous SSA treatment of median 26-month duration. A retrospective analysis was performed on prospectively maintained databases from 13 Italian NET-dedicated centres. Patients either underwent an increased intensity (dose) or density (dose frequency) of SSA therapy, and primary outcome measures were PFS (measured using the Kaplan-Meier method) and safety (by recording adverse effects). Increased treatment 'density' was achieved by shortening the normal interval between administration from 28 days to 14 or 21 days, while increased 'intensity' was achieved by a higher drug dose (lanreotide $180 \mathrm{mg}$, octreotide $60 \mathrm{mg}$ ), with normal 28-day cycles. The median duration of treatment was 16 months. It should be noted that $95 \%$ of patients $(n=133)$ were in the increased density group, with just $5 \%(n=7)$ receiving the increased intensity treatment, preventing any definitive intergroup comparisons. ${ }^{8}$

In general, $75.7 \%$ of patients achieved stable disease, and median PFS was found to be 31 months. The objective response rate, defined as the proportion of patients who achieved a complete or partial response, was $8.6 \%$. Of note was the fact that a significantly longer PFS of 57 months was associated with patients for whom the HD-SSA was a second-line treatment, compared with third- or further-line treatments, whose PFS was 22 months. On multivariate analysis, use of HD-SSAs as a third- or further-line treatment was independently associated with a higher risk of progression or death. A proposed explanation for this association was that consecutive courses of systemic therapies may lead to a progressive selection of aggressive, treatment-resistant clones. Interestingly, the data also showed there to be no significant difference in median PFS between participants of differing tumour grade. The authors attributed this to a low proliferation index in the series, with a median Ki-67 of $2 \%$, and an under-representation of high-end G2 tumours, obscuring any differences in response and survival between G1 and G2 tumours. With regards to the safety of HD-SSAs, these regimens were found overall to be well-tolerated: 21 patients (15\%) experienced some form of adverse effect, the majority of which were diarrhoea; 2 patients developed grade 3 biliary stone disease. ${ }^{8}$

Although these initial data look promising, one cannot form any firm conclusions concerning the superiority of the high-dose regimen, given the lack of a patient control group continuing conventional standard dose SSA (SD-SSA) therapy. This was most likely due to the ethical issue of continuing patients with progressive disease on a seemingly ineffective standard regimen, and provides a somewhat unavoidable limitation. This study relies on the assumption that if all participants exhibited progression on SD-SSA therapy prior to commencing their HD-SSA, and $75.7 \%$ subsequently achieved stable disease, then the treatment could be deemed effective. However, this is contingent on the imaging protocol being consistent before and after the switch, and the imaging intervals are not given, nor is it clear whether the imaging comparisons were undertaken and reviewed independently. This study is also limited by its retrospective nature and an inherent selection bias, in that patients treated with HD-SSAs in clinical practice tend to be those with more indolent NETS. Furthermore, the small size of the increased intensity arm $(n=7)$ prevented conclusive evaluation of this strategy, let alone any meaningful comparison between the two study arms.

It is important to note that some disparity exists concerning toxicity rates and PFS estimates between this study and the control arm of the phase III NETTER-1 trial, ${ }^{9}$ and these discrepancies cannot be overlooked. In the NETTER-1 trial, patients with well-differentiated, midgut NETS were mandated to have RECIST disease progression at baseline while receiving standard octreotide LAR therapy (20-30 mg every 3-4 weeks, for at least 12 weeks before randomisation). Patients randomised to the control arm were then treated with octreotide LAR $60 \mathrm{mg}$ every 4 weeks. This control population was found to have favourable baseline characteristics, including a predominance of G1 histopathology (72\%) and low rates of prior systemic therapy other than SSA (15\% molecular-targeted therapy, 12\% chemotherapy) - Lamberti and colleagues similarly had a predominantly low-grade study population. ${ }^{8}$ A computed tomography scan or magnetic resonance imaging was performed every 12 weeks to evaluate objective tumour response with central assessment.9 At the time of the data cut-off for the primary analysis, the median PFS was 8.4 months $(95 \%$ confidence interval [Cl] 5.8-9.1) in the control group, compared with a corresponding median of 31.0 months in the Lamberti study. Furthermore, toxicity of any grade was reported in $84 \%$ of the control arm population (33\% of which were grade 3 or 4 toxicity), mainly consisting of abdominal pain (26\%), diarrhoea (19\%) and fatigue (25\%). This is in stark contrast to the figure of $15 \%$ reported by Lamberti and colleagues. It is noted in the Lamberti study that the majority of patients received an increased dose density rather than dose intensity. Nevertheless, the markedly different PFS estimates and rates of toxicity in two seemingly low-grade populations on comparable HD-SSA regimens highlights the need for a randomised controlled trial to overcome the fundamental issues of confounding and bias.

It should be noted that the primary aim of the phase III NETTER-1 trial was actually to evaluate the efficacy and safety of peptide receptor radionuclide therapy (PRRT) in combination with SD-SSA, compared with that of HD-SSA alone.' PRRT treats neoplasms by delivering targeted radiotherapy to malignant NET cells expressing SSTRS. Strosberg and colleagues randomised patients to receive either a combination therapy of ${ }^{177}$ Lu-DOTATATE $(7.4 \mathrm{GBq})$ every 8 weeks plus best supportive care consisting of octreotide LAR (30 mg) every 4 weeks, or octreotide LAR (60 mg) every 4 weeks. ${ }^{9}$ The primary outcome measure was PFS. At 20 months, the ${ }^{177}$ Lu-DOTATATE group had an estimated PFS rate of $65.2 \%$ (95\% Cl 50.0-76.8), compared with $10.8 \%$ (95\% Cl 3.5-23.0) in the control group. While the Cls are fairly large, this is quite a notable result. Moreover, the respective objective response rates for each group were $18 \%$ ( ${ }^{177}$ Lu-DOTATATE) and 3\% (octreotide LAR). ${ }^{9}$ Subsequent analyses of the NETTER-1 trial have also confirmed a significant improvement in quality of life in patients treated with PRRT. ${ }^{10}$ The important decision therefore rests as to whether patients with G1/G2 disease advancing on SD-SSA should move first to HD-SSA or proceed straight to PRRT, or indeed other therapies such as molecular-targeted agents or chemotherapy. Individualised assessments will need to be made in each case, but in our opinion a move to HD-SSA initially is simplest, most convenient for the patient, and of relatively low cost. Of course, in patients with rapid progression or extensive disease, more intensive therapies may be required.

In summary, while there ideally remains a need for prospective, randomised controlled trials, these data are sufficiently positive for many of us to feel reassured that the switch to high-dose regimens in patients with progressive, low-grade disease is safe and likely beneficial, although the duration of benefit is yet to be clearly defined. In the meantime, we look forward to the results of a prospective phase ॥ study that is reportedly completed, but awaiting analysis and publication (CLARINET FORTE; ClinicalTrials.gov identifier: NCT02651987), looking at PFS in patients taking $120 \mathrm{mg}$ lanreotide autogel every 14 days, who previously experienced disease progression while on the standard $120 \mathrm{mg}$ 28-day regimen. This may strengthen the case for use of HD-SSAs in advanced NETs and highlight their potential to improve long-term outcomes for this challenging group of neoplasms. $]$ 
1. Wu J, Sun $C$, Li E, et al. Non-functional pancreatic neuroendocrine tumours: emerging trends in incidence and mortality. BMC Cancer. 2019;19:334.

2. Grozinsky-Glasberg S, Franchi G, Teng M, et al. Octreotide and the mTOR inhibitor RADO01 block proliferation and interact with the Akt-mTOR-p70S6K pathway in a neuroendocrine tumor cell line. Neuroendocrinology. 2008;87:168-81.

3. Strosberg J, Kvols L. Antiproliferative effect of somatostatin analogs in gastroenteropancreatic neuroendocrine tumors. World I Gastroenterol. 2010;16:2963-70.

4. Rinke A, Müller HH, Schade-Brittinger C, et al. Placebo-controlled, double-blind, prospective, randomized study on the effect of octreotide LAR in the control of tumor growth in patients with metastatic neuroendocrine midgut tumors: a report from the PROMID study group. J Clin Oncol. 2009;27:4656-63. 5. Caplin ME, Pavel M, Ćwikła JB, et al. Lanreotide in metastatic 2014;371:224-33.

6. Pavel $\mathrm{M}, \mathrm{O}^{\prime}$ Toole $\mathrm{D}$, Costa F, et al. ENETS consensus guidelines update for the management of distant metastatic disease of intestinal, pancreatic, bronchial neuroendocrine neoplasms (NEN) and NEN of unknown primary site. Neuroendocrinology. 2016;103:172-85.

7. Welin SV, Janson ET, Sundin A, et al. High-dose treatment with a long-acting somatostatin analogue in patients with advanced midgut carcinoid tumours. Eur J Endocrinol. 2004;151:107-12.
8. Lamberti G, Faggiano A, Brighi N, et al. Nonconventional doses of somatostatin analogs in patients with progressing well-differentiated neuroendocrine tumor. J Clin Endocrinol Metab. 2019:105:194-200.

9. Strosberg J, El-Haddad G, Wolin E, et al. Phase 3 trial of 177Lu-Dotatate for midgut neuroendocrine tumors. 177Lu-Dotatate for midgut neuroend

N Eng/J Med. 2017;376:125-35.
10. Strosberg J, Wolin E, Chasen B, et al. Health-related quality of life in patients with progressive midgut neuroendocrine tumors treated with ${ }^{177} \mathrm{Lu}$-dotatate in the phase III NETTER-1 trial. J Clin Oncol. 2018;36:2578-84. 